\title{
CORRESPONDENCE
}

\section{Favorable outcomes among neonates not separated from their symptomatic SARS-CoV-2-infected mothers}

Pediatric Research (2021) 90:8-11; https://doi.org/10.1038/s41390020-01226-3

\section{INTRODUCTION}

Coronavirus disease 2019 (COVID-19), resulting from infection with severe acute respiratory syndrome coronavirus 2 (SARS-CoV-2), can affect pregnant women. Their newborns are at a higher risk of prematurity and early separation from their mothers, who may subsequently require intensive care for their own health. ${ }^{1,2}$ Although neonates born of mothers infected with SARS-CoV-2 during pregnancy are seemingly vulnerable to infection, studies have found that they were not at a high risk for severe infection and were very rarely affected by COVID-19. ${ }^{1-6}$ The presence of virus by real-time reverse transcriptase polymerase chain reaction (RT-PCR) has been reported in newborns before $\mathrm{H} 12$ of life. ${ }^{1}$ In addition, antiviral immunoglobulin $M$ has been detected in newborns at birth, ${ }^{7}$ suggesting that mother-to-infant viral transmission may occur. ${ }^{8}$ To date, however, only one case of vertical transmission has been clearly demonstrated. ${ }^{9}$ In several cases of early neonatal infection, postnatal contamination cannot be excluded. ${ }^{10}$ Moreover, only one case report found that the virus could pass from mother to infant through the mother's breast milk. ${ }^{11}$

Despite these findings, international or national guidelines on the management of newborns of mothers suspected or positive for COVID-19, including Chinese consensus guidelines and guidelines of the American Academic of Pediatrics, have recommended at least temporary separation of mother and newborn as a precaution. ${ }^{12,13}$ In contrast, other guidelines, ${ }^{14}$ including those of the French Society of Neonatology, ${ }^{15,16}$ do not recommend systematic separation. Most of these guidelines consistently support breastfeeding if the mother wishes it. ${ }^{14,17}$ All guidelines, however, recommend isolating these newborns for 14 days.

The Alsace region was one of the main sites of the COVID-19 pandemic in France in March 2020. The two level III perinatal centers in Alsace implemented similar guidelines, with no systematic mother-infant separation, breastfeeding support, and a structured follow-up during the first month of life. The main objective of this study was to assess the safety of the current management. The secondary objective was to evaluate the need for re-hospitalization during the follow-up period.

\section{METHODS}

This retrospective study evaluated all term and near-term neonates born to mothers at Strasbourg University and Mulhouse Hospitals in Alsace, France confirmed as having COVID-19 during their last 2 weeks of pregnancy between March 15 and April 24,
2020. After birth, mothers and newborns were separated only when their medical status made this necessary. Skin-to-skin contact and breastfeeding required specific hygienic measures. Mothers had to wear surgical face masks, disinfect their hands, and wash their nipples with odorless soap. Direct breastfeeding, indirect breastfeeding through expressed breast milk, and formula feeding were at the mother's preference. When mothers were sleeping, cradles had to be placed at least $2 \mathrm{~m}$ away from their beds. Visits from others were not permitted. Mothers were able to access their hospitalized neonates securely as soon as allowed by their medical status. Nasopharyngeal and anal swabs were obtained from each neonate at birth and on day 3, as well as weekly from neonates requiring a prolonged hospital stay, and breast milk samples were obtained on day 3 and/or day 7 . The presence of SARS-CoV-2 RNA in these samples was assessed by inhouse RT-PCR, with an assay sensitivity of about 10 copies/ reaction (https://www.who.int/docs/default-source/coronaviruse/ real-time-rt-pcr-assays-for-the-detection-of-sars-cov-2-institutpasteur-paris.pdf?sfvrsn $=3662 \mathrm{fcb} 6 \_2$ ), using primers and probes targeting two regions on the RdRp gene and specific to SARSCoV2.

Neonates were discharged after day 3 of life through hospitalassisted neonatal home care coordinated by a pediatrician. Home care included daily planned visits by a midwife until day 8 and every 2 days for breastfed infants or at days 10 and 14 for nonbreastfed infants. At each visit, the newborn was weighed, and feeding methods and signs of illness were noted. Each newborn was examined in person by their pediatrician on day 14. Parents were able to call a neonatologist at any time, with systematic teleconsultation on days $8,14,21$, and 28 . The neonatologist provided advice, answered questions, and checked the medical status of the neonate, the mother, and the family. The study was approved on 14 May 2020 by the local ethics committee of the Strasbourg Faculty of Medicine (CE-2020-86), and all parents provided written informed consent.

\section{RESULTS}

Twenty-six mother-neonate dyads were included (Table 1). Only one preterm neonate required endotracheal intubation and surfactant administration. He was rapidly extubated after neonatal intensive care unit (NICU) admission and required no additional respiratory support. Three other neonates were admitted to the NICU, one of gestational age (GA) 34 weeks, one of GA 35 weeks with respiratory distress, and a full-term neonate with hemolytic anemia. Three neonates were never placed in the same room as their mothers, although all mothers had free access to their newborns. Most of the neonates were breastfed and were supplemented with artificial milk (formula) or pumped breast milk. Median hospital stay was 4 days (range, 3-18 days). RT-PCR showed that one anal swab, obtained on day 3 from a full-term, asymptomatic neonate, was positive for SARS-CoV-2 RNA. How- 
Table 1. Perinatal characteristics of the 26 mothers with COVID-19 and their neonates.

Maternal characteristics $(n=26)$

Age, years, mean (SD)

Comorbidity before pregnancy ${ }^{a}$

Complication of pregnancy ${ }^{b}$

Time, days, median (IQR) from symptom appearance to delivery

Vaginal delivery

Cesarean section

Fever during labor

Respiratory signs

Severe acute respiratory distress syndrome

Neonatal characteristics at birth $(n=26)$

Gestational age, weeks, mean (SD)

Weight, g, median (IQR)

Small for gestational age ${ }^{c}$

APGAR score at $5 \mathrm{~min}>7$

Resuscitation with endotracheal intubation

Delivery room outcomes

Breastfeeding

Skin-to-skin contact

Skin-to-skin contact or breastfeeding

Positive SARS-CoV-2 RT-PCR ${ }^{\mathrm{d}}$ at day 0 or day 1

Maternity ward and neonatal unit outcomes

Hospitalization in the NICU

Hospitalization in the conventional neonatal unit

Because of prematurity

Because of maternal medical status

Feeding method

Exclusive direct breastfeeding

Direct breastfeeding and expressed breast milk or formula milk

Exclusive formula milk

Clinical symptoms

Hyperthermia

$30.6(7.9)$

3 (11.5)

$16(61.5)$

$10(38.5)$

8 (30.7)

16 (61.5)

2 (7.6)

Nasopharyngeal congestion

Respiratory distress

Vomiting or diarrhea

Neurological symptoms

Jaundice

Neonate positive for SARS-CoV-2 ${ }^{d}$ by RT-PCR after day 2

Values are number (\%) unless otherwise stated.

$R T-P C R$ reverse transcription polymerase chain reaction, $S D$ standard deviation, $I Q R$ interquartile range.

aHypertension, obesity, diabetes, or asthma.

byypertension, preeclampsia, diabetes, or cholestasis.

'Birth weight below the 10th percentile.

${ }^{d}$ RT-PCR for SARS-CoV-2 of nasopharyngeal and stool or anal swabs in 25 neonates.

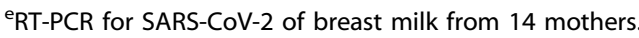

ever, nasopharyngeal swabs obtained from this newborn on days 0 and 3 , an anal swab obtained on day 0 , and a stool sample obtained on day 3 were all negative. This infant developed no clinical symptoms later. All other samples from neonates and all breast milk samples were negative for SARS-CoV-2 RNA.

Outcomes 1 month after hospital discharge are summarized in Table 2. None of the newborns was re-hospitalized during their first month of life. One full-term newborn was brought to the pediatric emergency room on day 9 for mild nasal congestion without any other pathological signs. Examination by a midwife on days 10,12, and 14 showed rapid disappearance of the respiratory symptoms. No other newborn required special care.

\section{DISCUSSION}

We evaluated the risks and benefits of a strategy to deal with newborns of mothers infected with SARS-CoV-2, mainly during their last week of pregnancy. This strategy involved preservation of continuous mother-infant proximity with specific hygienic measures, breast milk as the main source of feeding, early discharge with home isolation, and a structured follow-up with hospital-assisted home care. Evaluation of 1-month outcomes in these 26 neonates found that this strategy was feasible and safe. Except for one infant, who was transiently positive for SARS-CoV-2 RNA but had no clinical signs, none of these newborns was infected, had adverse outcomes, or presented with any virusrelated clinical symptoms until age 1 month, with none requiring hospital readmission.

This study had some limitations, including the relatively small number of infants. In addition, repeated and systematic viral tests were not performed, especially on breast milk, until the end of the study period. However, this study included all newborns at two institutions who met the inclusion criteria during the COVID-19 pandemic and with detailed clinical data on their hospital care and full follow-up. These results are in accordance with the very low incidence of infection in newborns of infected mothers in a national cohort of the United Kingdom, in which guidelines for the postnatal management of infants of infected mothers included keeping the mother and infant together and encouraging breastfeeding with strict infection control measures from birth. ${ }^{1}$ Our data add more information on the perinatal viral status and clinical follow-up until the end of the neonatal period of neonates cared for using a similar strategy. Our findings also support similar guidelines of various countries and health care societies ${ }^{17}$ and recently reported data. ${ }^{6}$ In our study, with a high proportion of infants fed with breast milk, we found no evidence for horizontal transmission. Breastfeeding may protect against the horizontal transmission of SARS-CoV-2, as specific antibodies against this virus have been found in the breast milk of a COVID-19-infected mother. ${ }^{18}$ This hypothesis appears consistent with the protective effect of breastfeeding against respiratory tract infections in infancy. ${ }^{19}$

Our results support early postnatal proximity, despite many mothers worldwide being separated from their newborn infants during the COVID-19 pandemic. Early separation strategies have been reported to have deleterious mid- and long-term effects and early separation is discouraged. ${ }^{20}$ Infant and family-centered care can be maintained during the COVID-19 pandemic with preservation of the many benefits provided by early skin-to-skin contact, including breastfeeding when desired. ${ }^{17}$ Lack of separation may also reduce stresses on parents during the pandemic, especially when fathers, in many settings, have restricted access to mothers and newborn infants around birth. ${ }^{21,22}$

The combination of hospital-assisted neonatal home care and telehealth, which should be used whenever possible, appears fully compatible with family-centered care. It seems to allow secure case management, safe follow-up, and the maintenance of hygiene barriers at home, reducing postnatal horizontal transmission. Although we did not observe any apparent postnatal infection, a possible complication rarely described, ${ }^{10}$ we cannot 
Table 2. Neonatal outcomes during home follow-up.

\begin{tabular}{lccc}
\hline Outcomes of discharged neonates & From day 4 to day $8(n=20)$ & From day 9 to day $14(n=25)$ & From day 14 to day $28(n=26)$ \\
\hline Absence of any clinical symptoms & $20(100)$ & $24(96)$ & $25(96.2)$ \\
Nasal congestion & $0(0)$ & $1(4,0)$ & $0(0)$ \\
Diarrhea & $0(0)$ & $0(0)$ & $1(3.8)$ \\
Weight gain, g/day, median (IQR) & $33(39.3)$ & $40(24.8)$ & - \\
Emergency consultation & $0(0)$ & $1(4,0)$ & $0(0)$ \\
Protocol-planned consultation & $17(85.0)$ & $25(100)$ & $10(10)$ \\
Parental calls & $0(0)$ & $9(0)$ & $8(30)$ \\
Exclusive breastfeeding & $10(50.0)$ & $12(48.0)$ & $12(46.1)$ \\
Breastfeeding and expressed breast milk or & $8(40.0)$ & $1(4.0)$ & $0(0)$ \\
formula milk & $0(0)$ & $3(12.0)$ & $6(23.0)$ \\
Expressed breast milk and formula milk & $2(10.0)$ &
\end{tabular}

Values are number (\%) unless otherwise stated.

$I Q R$ interquartile range.

fully rule out the possibility of asymptomatic infection through postnatal contact, as we did not test these infants for SARS-CoV-2 infection at age 1 month. To date, however, no late postnatal mother-infant transmission has been reported after 1 month of age.

\section{CONCLUSION}

Even during the COVID-19 pandemic, safely maintaining familycentered perinatal care and continuing the promotion of bonding between neonates and their SARS-CoV-2-positive mothers appear possible, as these newborns are very rarely infected and, if infected, show only mild symptoms. Population-based studies, with longer postnatal follow-ups, are warranted to fully support this strategy. The impact of this approach should also be evaluated in preterm infants born to infected mothers.

\section{ACKNOWLEDGEMENTS}

We are very grateful to the participating neonates and their parents. This study was funded by the Strasbourg University Hospital.

\section{AUTHOR CONTRIBUTIONS}

A.M. collected and analyzed the perinatal data and the literature. He drafted the first version of the manuscript and contributed substantially to the revised version. I.L. organized the follow-up, collected and analyzed the neonatal and home care data, and revised the manuscript. A.D.-S., O.M., C.L., V.P.-K., and F.D.M. collected, analyzed, and interpreted the perinatal data and revised the manuscript. H.D. collected, analyzed, and interpreted the virological data and revised the manuscript. S.F.-K. and P.D. conceived and designed the study and revised the manuscript for important intellectual content. P.K. conceived and designed the study, analyzed the data, had substantial implication in the writing and the revision of the manuscript, and gave final approval of the version to be published. All authors approved the final manuscript.

\section{ADDITIONAL INFORMATION}

Competing interests: The authors declare no competing interests.

Patient consents: Parental consent was required and obtained.

Publisher's note Springer Nature remains neutral with regard to jurisdictional claims in published maps and institutional affiliations.
Antoine Martenot ${ }^{1}$, Imad Labbassi ${ }^{1}$, Amélie Delfils-Stern ${ }^{2}$,

Oscar Monroy ${ }^{1}$, Claire Langlet ${ }^{1}$, Valérie Pichault-Klein ${ }^{2}$, Héloise Delagreverie ${ }^{3}$, Fanny De Marcillac ${ }^{4}$, Samira Fafi-Kremer ${ }^{3,5}{ }^{\prime}$, Philippe Deruelle ${ }^{4}$ and Pierre Kuhn ${ }^{1,6}$

${ }^{1}$ Department of Neonatology, University Hospital of Strasbourg, Strasbourg, France; ${ }^{2}$ Department of Neonatology, Hospital of Mulhouse, Mulhouse, France; ${ }^{3}$ Medical Virology Laboratory, University Hospital of Strasbourg, Strasbourg, France; ${ }^{4}$ Department of Obstetrics, University Hospital of Strasbourg, Strasbourg, France; ${ }^{5}$ Strasbourg University, INSERM, IRM UMR-S 1109, 67000 Strasbourg, France and ${ }^{6}$ Strasbourg University, Institut des Neurosciences Cellulaires et Intégratives, CNRS UPR 3212, Strasbourg, France Correspondence: Pierre Kuhn (pierre.kuhn@chru-strasbourg.fr)

\section{REFERENCES}

1. Knight, M. et al. Characteristics andoutcomes of pregnant women admitted to hospital with confirmed SARS-CoV-2 infection in UK: national population based cohort study. BMJ 369, m2107 (2020).

2. Sentilhes, L. et al. COVID-19 in pregnancy was associated with maternal morbidity and preterm birth. Am. J. Obstet. Gynecol. https://doi.org/10.1016/j.ajog.2020.06.022 (2020).

3. Ludvigsson, J. F. Systematic review of COVID-19 in children shows milder cases and a better prognosis than adults. Acta Paediatr. 109, 1088-1095 (2020).

4. Perrone, S. et al. Report of a series of healthy term newborns from convalescent mothers with COVID-19. Acta Biomed. 91, 251-255 (2020).

5. Perlman, J., Oxford, C., Chang, C., Salvatore, C. \& Di Pace, J. Delivery room preparedness and early neonatal outcomes during COVID 19 pandemic in New York City. Pediatrics 146, e20201567 (2020).

6. Salvatore, C. M. et al. Neonatal management and outcomes during the COVID-19 pandemic: an observation cohort study. Lancet Child Adolesc. Health 4, 721-727 (2020).

7. Dong, L. et al. Possible vertical transmission of SARS-CoV-2 from an infected mother to her newborn. JAMA 323, 1846-1848 (2020).

8. Chen, $\mathrm{H}$. et al. Clinical characteristics and intrauterine vertical transmission potential of COVID-19 infection in nine pregnant women: a retrospective review of medical records. Lancet 395, 809-815 (2020).

9. Vivanti, A. J. et al. Transplacental transmission of SARS-CoV-2 infection. Nat. Commun. 11, 3572 (2020).

10. Buonsenso, D. et al. Neonatal late onset infection with severe acute respiratory syndrome coronavirus 2. Am. J. Perinatol. https://doi.org/10.1055/s-0040-1710541 (2020).

11. Gross, R. et al. Detection of SARS-CoV-2 in human breastmilk. Lancet 395, 1757-1758 (2020).

12. Chen, D. et al. Expert consensus for managing pregnant women and neonates born to mothers with suspected or confirmed novel coronavirus (COVID-19) infection. Int. J. Gynaecol. Obstet. 149, 130-136 (2020). 
13. Puopolo, K. M., Hudak, M. L., Kimberlin, D. W. \& Cummings, J. American Academy of Pediatrics. Management of infants born to mothers with COVID-19. https:// downloads.aap.org/AAP/PDF/COVID\%2019\%20Initial\%20Newborn\%20Guidance. pdf (2020).

14. World Health Organization. Breastfeeding advice during the COVID-19 outbreak. http://www.emro.who.int/nutrition/nutrition-infocus/breastfeeding-adviceduring-covid-19-outbreak.html (2020).

15. Vivanti, A. J. et al. Post-natal follow-up for women and neonates during the COVID-19 pandemic: French National Authority for Health recommendations. J. Gynecol. Obstet. Hum. Reprod. https://doi.org/10.1016/j.jogoh.2020.101805 (2020).

16. Société française de Néonatalogie. Société française de Pédiatrie. Propositions de la société française de néonatalogie et de la société française de pédiatrie concernant les nouveau-nés dans le contexte d'épidémie à covid-19. https://f4ed7074-25ed-461c8cf3-ddd4393f43e2.filesusr.com/ugd/d8ff38_bc9913a3008a4cf7bd90f0e74832eb18. pdf (2020).
17. Tscherning, C., Sizun, J. \& Kuhn, P. Promoting attachment between parents and neonates despite the COVID-19 pandemic. Acta Paediatr. https://doi.org/10.1111/ apa.15455 (2020).

18. Dong, Y. et al. Antibodies in the breast milk of a maternal woman with COVID-19. Emerg. Microbes Infect. https://doi.org/10.1080/22221751.2020.1780952 (2020).

19. Duijts, L., Ramadhani, M. K. \& Moll, H. A. Breastfeeding protects against infectious diseases during infancy in industrialized countries. A systematic review. Matern. Child Nutr. 5, 199-210 (2009).

20. Tomori, C., Gribble, K., Palmquist, A. E. L., Ververs, M. T. \& Gross, M. S. When separation is not the answer: breastfeeding mothers and infants affected by COVID-19. Matern. Child Nutr. https://doi.org/10.1111/mcn.13033 (2020).

21. Stuebe, A. Should infants be separated from mothers with COVID-19? First, do no harm. Breastfeed. Med. 15, 351-352 (2020).

22. Lista, G. \& Bresesti, I. Fatherhood during the COVID-19 pandemic: an unexpected turnaround. Early Hum. Dev. https://doi.org/10.1016/j.earlhumdev.2020.105048 (2020). 